\title{
Flux Flushing in Superconducting Niobium Films
}

\author{
M. C. de Andrade, S. Berggren, B. J. Taylor, A. M. Leese de Escobar \\ SPAWAR Systems Center Pacific \\ San Diego, CA USA \\ Email: marcio.deandrade@navy.mil
}

\begin{abstract}
Flux trapping in superconducting devices has shown to be detrimental to the consistent operation of superconducting electronics (SCE). Approaches to improve reliability of SCE components have focused on introduction of flux trap regions and highly elaborate degaussing procedures. Nevertheless, a controlled and reproducible method to assure the elimination of trapped flux on SCE devices remains elusive. A substantial body of work on artificial defects utilizing the so called ratchet effect has demonstrate limited control of the magnetic vortices in niobium films. These early attempts to control the spurious vortices distribution have been limited to small geometrical regions having no practical effect on improving SCE devices operational parameters. In this paper, we report simulations and propose an improved method utilizing the ratchet effect that can be extended to physical sizes compatible to existing fabrication techniques of SCE devices.
\end{abstract}

Index Terms-flux trapping; ratchet effect; superconducting electronics; vortices

\section{INTRODUCTION}

Superconducting electronic devices, in particular the ones that use Josephson junction as the building block for its logic structure, can be rendered inoperable due to the likelihood of magnetic flux trapping [1]. Typically present in the superconducting ground plane, the trapped flux generates internal noise as well as dissipates energy. A combination of pinning sites have been used to model the effects of controlling the trapped flux, including using points [2]-[4] or the geometry of a sawtooth [5]-[8] to create an artificial potential [9]. The application of an AC current to act as an external force is applied to the system to examine how to control, and set into motion, the trapped vortices [10].

In this work, we look at specific results for when an $\mathrm{AC}$ current is applied to the asymmetrical pinning potential, which takes advantage of the 'ratchet effect'. We extend the original theory from its restricted small areas of around $2 \times 2 \mu \mathrm{m}^{2}$, to a large $10 \times 10 \mathrm{~mm}^{2}$, typical of state of the art devices. We show that this topology, associated with a procedural method, is able to clear the vortices from an area of $10 \times 10 \mathrm{~mm}^{2}$. Additionally, we discuss the behavior of the vortices once the AC Current is turned off, and show that the vortices seem to not return to the initial state, permanently clearing the studied area.

\section{MODEL}

In this section we will describe the model, go over some of the simulation details, and then convert the parameters to experimental values.

\section{A. Theoretical Description}

As described in [2], the velocity of the vortex $i$ is related to the total force $\boldsymbol{f}_{i}$ acting on vortex $i$ divided by the viscosity $\eta$.

$$
\boldsymbol{v}_{i}=\frac{\boldsymbol{f}_{i}}{\eta}=\frac{\boldsymbol{f}_{i}^{v v}+\boldsymbol{f}_{i}^{u}+\boldsymbol{f}_{d}}{\eta},
$$

where $f_{i}^{v v}$ is the force of the vortices on each other, $f_{i}^{u}$ is the force generated by the pinning features (here a periodic asymmetrical potential) and $f_{d}$ is the driving force. The vortex-vortex interaction can be modeled

$$
\boldsymbol{f}_{i}^{v v}=\sum_{j=1}^{N_{v}} f_{0} K_{1}\left(\frac{\left|\boldsymbol{r}_{i}-\boldsymbol{r}_{j}\right|}{\lambda}\right) \hat{\boldsymbol{r}}_{i j},
$$

where $\boldsymbol{r}_{i}$ and $\boldsymbol{r}_{j}$ are the locations of vortices $i$ and $j$ respectively, $N_{v}$ is the number of vortices, $\hat{\boldsymbol{r}}_{i j}=\left(\boldsymbol{r}_{i}-\boldsymbol{r}_{j}\right) /\left|\boldsymbol{r}_{i}-\boldsymbol{r}_{j}\right|$, $K_{1}$ is a modified Bessel function, $\lambda$ is the penetration depth and $f_{0}=\frac{\Phi_{0}^{2} d}{2 \pi \lambda^{3} \mu_{0}}$. In $f_{0}, \Phi_{0}=\frac{h}{2 e}$ is the elementary flux quantum, $d$ is the thickness and $\mu_{0}$ is the permeability of free space.

The potential force is an asymmetrical sawtooth function that can be seen in Fig. 1. It can be modeled as

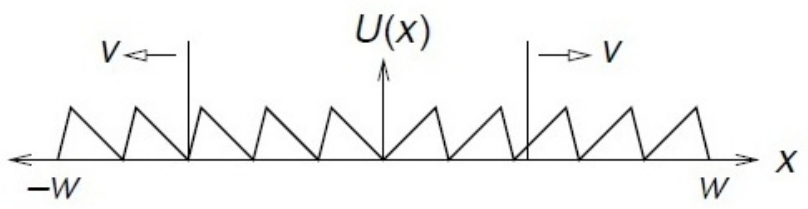

Fig. 1. The side view of the asymmetrical sawtooth potential from [5]

$$
\boldsymbol{f}_{i}^{u}=\left\{\begin{array}{ll}
\hat{\boldsymbol{x}} & \text { if } 0 \leq\left(\boldsymbol{r}_{i} \hat{\boldsymbol{x}} \bmod 1\right)<1 / 3 \text { and } \boldsymbol{r}_{i} \hat{\boldsymbol{x}}<\frac{x_{\max }}{2} \\
-\frac{1}{2} \hat{\boldsymbol{x}} & \text { if } 1 / 3 \leq\left(\boldsymbol{r}_{i} \hat{\boldsymbol{x}} \bmod 1\right)<1 \text { and } \boldsymbol{r}_{i} \hat{\boldsymbol{x}}<\frac{x_{\max }}{2} \\
-\frac{1}{2} \hat{\boldsymbol{x}} & \text { if } 0 \leq\left(\boldsymbol{r}_{i} \hat{\boldsymbol{x}} \bmod 1\right)<2 / 3 \text { and } \boldsymbol{r}_{i} \hat{\boldsymbol{x}}>\frac{x_{\max }}{2} \\
\hat{\boldsymbol{x}} & \text { if } 2 / 3 \leq\left(\boldsymbol{r}_{i} \hat{\boldsymbol{x}} \bmod 1\right)<1 \text { and } \boldsymbol{r}_{i} \hat{\boldsymbol{x}}>\frac{x_{\max }}{2}
\end{array},\right.
$$

where $x_{\max }$ is the size of the film in the $x$-direction.

\section{B. Simulation Details}

For the results shown in the next section $f_{d}$ is an AC current of magnitude 1.0 which has a period of $4000 d t(d t=0.001)$. All the distances are measured in units of $\lambda$, forces are measured in units of $f_{0}$, time is measured in units of $\frac{\eta d}{f_{0}}$ and 
fields are in units of $\frac{\Phi_{0}}{\lambda^{2}}$. In Eq. (1) $\eta$ is set to 1.0 and in Eq. (2) the modified Bessel function has a cutoff at $6 \lambda$ since the force is negligible for distances greater, and becomes constant for less than $0.1 \lambda$ to remove the logarithmic divergence issue.

\section{Experimental Values}

Throughout this simulation, in order to better associate the simulation parameters with experimental values, we used the following conversions:

1) Value of $f_{0}$ : The parameter $f_{0}$ is

$$
f_{0}=\frac{\Phi_{0}^{2} d}{2 \pi \lambda^{3} \mu_{0}},
$$

where $d$ is the film thickness and $\mu_{0}$ is the permeability of free space:

$$
\begin{aligned}
d & \approx 1000 \AA=1 \times 10^{-7} \mathrm{~m} \\
\mu_{0} & =4 \pi \times 10^{-7} \mathrm{~N} / \mathrm{A}^{2} .
\end{aligned}
$$

Using these $f_{0}$ can also be written

$$
f_{0}=\frac{\Phi_{0}^{2}}{4 \pi^{2} \lambda^{3}} .
$$

To determine $f_{0}$ we also need to know values for $\Phi_{0}$ and $\lambda$, which are

$$
\begin{aligned}
\Phi_{0} & =\frac{h}{2 e}=2.067834 \times 10^{-15} \mathrm{~Wb}\left(\text { or } \mathrm{Tm}^{2}\right) \\
\lambda & =45 \mathrm{~nm}=4.5 \times 10^{-8} \mathrm{~m} .
\end{aligned}
$$

Plugging into Eq. (4) gives

$$
\begin{aligned}
f_{0} & =\frac{\left(2.067834 \times 10^{-15} \mathrm{Tm}^{2}\right)^{2}\left(1 \times 10^{-7} \mathrm{~m}\right)}{2 \pi\left(4.5 \times 10^{-8} \mathrm{~m}\right)^{3}\left(4 \pi \times 10^{-7} \frac{\mathrm{N}}{\mathrm{A}^{2}}\right)} \\
& =5.943 \times 10^{-10} \mathrm{~N} .
\end{aligned}
$$

2) Film size: A common film size simulated in the references was $36 \lambda$ square. Using the penetration depth for Niobium we can approximate this to be

$$
36 \lambda \times 45 \mathrm{~nm}=1.62 \mu \mathrm{m} .
$$

To simulate a $10 \mathrm{~mm}$ by $10 \mathrm{~mm}$ film, we need to look at an area $222222 \lambda$ by $222222 \lambda$ since

$$
\frac{10 \mathrm{~mm}}{4.5 \times 10^{-5} \mathrm{~mm}}=222222 .
$$

3) Time Constant: The simulations use a normalized time $\tau$ which is related to $t$ by the equation

$$
t=\frac{\eta d}{f_{0}} \tau \text {. }
$$

The viscosity $\eta$ is

$$
\eta=\frac{\Phi_{0}^{2} d}{2 \pi \xi^{2} \rho_{N}},
$$

where $\xi=3.8 \times 10^{-8} \mathrm{~m}$ is the coherence length and $\rho_{N}$ is the normal state resistivity. To find a value for $\rho_{N}$ we notice that $\rho_{273 \mathrm{~K}}=1.45 \times 10^{-7} \Omega \mathrm{m}$ and that there is a $10^{4}$ decrease as
$T \rightarrow 0 \mathrm{~K}$ [11], so we assume $\rho_{N}=1.45 \times 10^{-11} \Omega \mathrm{m}$. Using $d$ from Eq. (3) and $\Phi_{0}$ from Eq. (4) results in

$$
\begin{aligned}
\eta & =\frac{\left(2.067834 \times 10^{-15} \mathrm{Tm}^{2}\right)^{2}\left(1 \times 10^{-7} \mathrm{~m}\right)}{2 \pi\left(3.8 \times 10^{-8} \mathrm{~m}\right)^{2}\left(1.52 \times 10^{-11} \Omega \mathrm{m}\right)} \\
& =3.2502 \times 10^{-12} \frac{\mathrm{kg}}{\mathrm{s}} .
\end{aligned}
$$

For Niobium with $d$ from Eq. (3), $f_{0}$ from Eq. (5) and $\eta$ from Eq. (6), we determine $\frac{\eta d}{f_{0}}$ to be

$$
\begin{aligned}
\frac{\eta d}{f_{0}} & =\frac{\left(1 \times 10^{-7} \mathrm{~m}\right)\left(3.2502 \times 10^{-12} \frac{\mathrm{kg}}{\mathrm{s}}\right)}{5.943 \times 10^{-10} \mathrm{~N}} \\
& =5.469 \times 10^{-10} \tau \mathrm{s}=0.5469 \mathrm{~ns} .
\end{aligned}
$$

The time range used for the smaller sized film simulations was from 0 to 200 with a step size of 0.001 . Using the time constant in Eq. (7) this equates to is 0 to $109.38 \mathrm{~ns}$ with a step size $0.5469 \mathrm{ps}$. The time it takes to clear a $10 \mathrm{~mm}$ by $10 \mathrm{~mm}$ size square of superconducting material is 400000 timesteps or

$$
400000 \times 0.5469 \mathrm{~ns}=218.76 \mu \mathrm{s} .
$$

4) Frequency and Amplitude of the AC current: The AC current is a square wave switching every 2000 time steps, has a period of

$$
T=4000 \times 0.5469 \mathrm{ps}=2.1876 \mathrm{~ns},
$$

and frequency of

$$
f=\frac{1}{T}=457.12196 \mathrm{MHz}
$$

Using $f_{0}$ from Eq. 5, the amplitude of the $\mathrm{AC}$ current is determined to be

$$
1.0 f_{0}=5.943 \times 10^{-10} \mathrm{~N} .
$$

\section{RESUlTS}

Initially we choose a random distribution of vortices for which 4000 time steps were simulated with $\boldsymbol{f}_{d}=0$ was used to obtain the initial conditions. An example of a $100 \lambda$ by $100 \lambda$ film with a sawtooth pattern containing 100 vortices is seen in Fig. 2 on the left. The plot on the right shows the path the vortices took when subjected to an AC current of amplitude
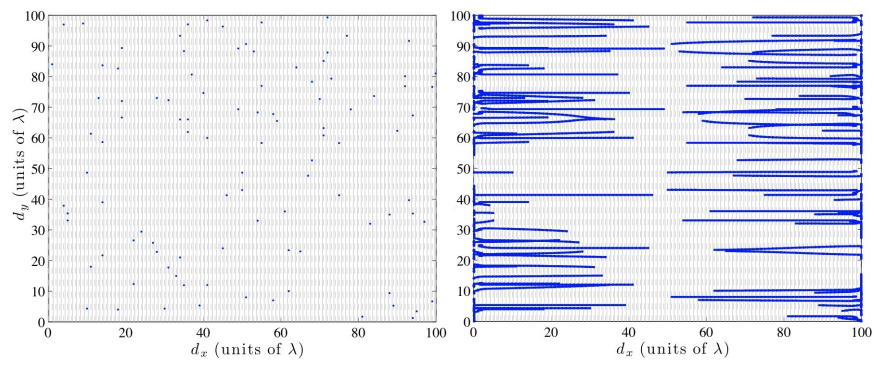

Fig. 2. Left: The starting locations of the vortices on a $100 \lambda$ by $100 \lambda$ film with a sawtooth pattern containing 100 vortices. Right: The path the vortices took when subjected to an AC current of amplitude 1.0, sign alternating every 2000 time steps. 
1.0, sign alternating every 2000 time steps. All the vortices move towards the closest side in the $x$-direction.

After 200000 time steps of size 0.001 the vortices are all crowded along the edge of the film and the center is empty, see Fig. 3. This means that the AC current with the asymmetrical potential (which takes advantage of the Ratchet effect removed the trapped flux in the film, which is what we want to happen.

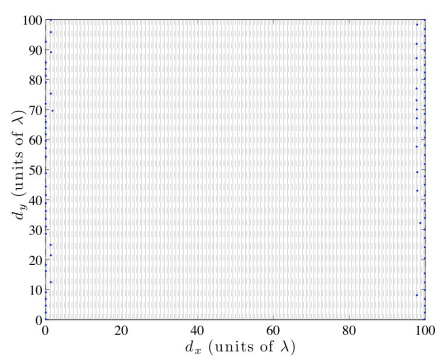

Fig. 3. Vortex locations on a $100 \lambda$ by $100 \lambda$ film with a sawtooth pattern containing 100 vortices after being subjected to an AC current, with amplitude 1.0 and period 4000 time steps, for 200000 time steps of size 0.001 .

If we turn off the $\mathrm{AC}$ current the path the vortices take in 200000 time steps of size 0.001 can be seen in on the left in Fig. 4. The right image is the final location of the vortices. We did this to explore what happens when the current stops, do the vortices flood the film immediately or do they stay away? The answer is, for this case, they all stayed at the edges in pretty much the same place.
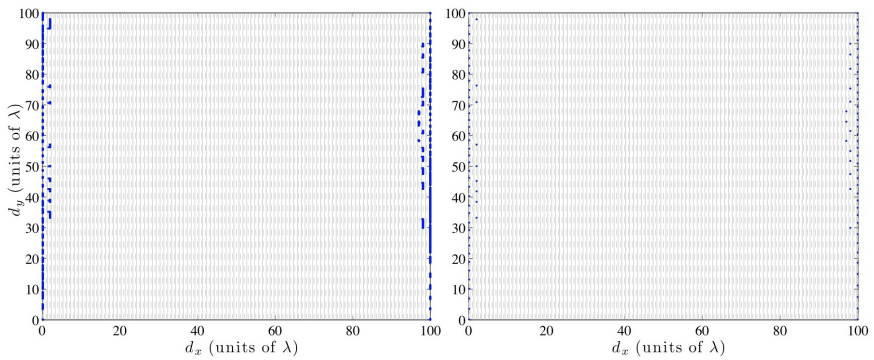

Fig. 4. The effects of turning off the AC current. Left: Path the vortices took Right: the final locations after the AC current being turned off for 200000 time steps.

Increasing the size of the film to $10 \mathrm{~mm}$ by $10 \mathrm{~mm}$ allows us to determine if the $\mathrm{AC}$ current acting on the asymmetrical sawtooth potential is able to clear a realistic area of material and how long it takes. The picture in Fig. 5 is the 10 vortices at the time 0 . The right image is the final location of the vortices after 400000000 time steps of size 0.001 when subjected to an AC current of amplitude 1.0, sign alternating every 2000 time steps. All the vortices are at the edge of the $10 \mathrm{~mm}$ by $10 \mathrm{~mm}$ film.

\section{CONCLUSION}

In this study we determined that an AC current applied to an asymmetrical sawtooth pattern is able to clear a realistic sized film, though it took many time steps. We also determined that once the AC current is turned off, the vortices all stayed at the edges if the film in pretty much the same place rather than returning to where they came from. The simulations were done with a low density of vortices to demonstrate these concepts. Increasing the number of vortices exponentially increases the simulation time. When there are very few vortices in a large area the vortex-vortex interaction is minimal. Further work into higher densities of vortices on realistic size wafers would be beneficial, especially to analyze the effects of vortex-vortex interactions on the effects of the AC current being turned off.

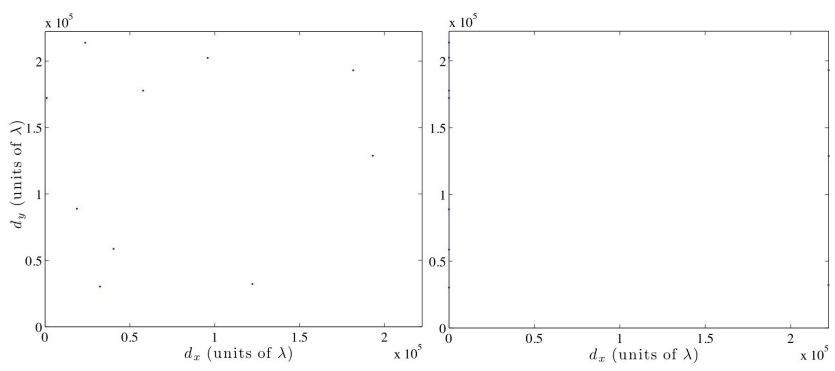

Fig. 5. Start locations (left) and end locations (right) of 10 vortices in a $10 \mathrm{~mm}$ by $10 \mathrm{~mm}$ film when subjected to an AC current of amplitude 1.0, sign alternating every 2000 time steps.

\section{ACKNOWLEDGMENT}

We acknowledge the support of Tactical SigInt Technology Program, and the financial support from the SSC Pacific In-house Laboratory Independent Research program and the Office of Naval Research. We are grateful for the invaluable early discussions with C. Reichhardt.

\section{REFERENCES}

[1] R. P. Robertazzi, I. Siddiqi, and O. A. Mukhanov, "Flux trapping experiments in single flux quantum shift registers," IEEE Trans. Appl. Supercond., vol. 7, no. 2, pp. 3164-3167, Jun 1997.

[2] C. Reichhardt, C. J. Olson, and F. Nori, "Nonequilibrium dynamic phases and plastic flow of driven vortex lattices in superconductirs with periodic arrays of pinning sites," Physical Review B, vol. 58, no. 10, pp. 6534-6564, Sep. 1998.

[3] C. J. Olson, C. Reichhardt, and F. Nori, "Nonequilibrium dynamic phase diagram for vortex lattices," Physical Review Letters, vol. 81, no. 17, pp. 3757-3760, Oct. 1998.

[4] C. Reichhardt, C. J. Olson, and F. Nori, "Dynamic phases of vorticies in superconductors with periodic pinning," Physical Review Letters, vol. 78, no. 13 , pp. 2658-2651, Mar. 1997.

[5] C. S. Lee, B. Jankó, I. Derényi, and A. L. Barabási, "Reducing vortex density in superconductors using the 'rachet effect'," Letters to Nature, vol. 400, pp. 337-340, Jul. 1999.

[6] Q. Lu, C. J. Olson Reichhardt, and C. Reichhardt, "Reversible vortex ratchet effects and ordering in superconductors with simple asymmetric potential arrays," Physical Review B, vol. 75, pp. 054502 1-9, 2007.

[7] W. Gillijns, A. V. Sihanek, V. V. Moshchalkov, C. J. Olson Reichhardt, and C. Reichhardt, "Origin of reversed vortex ratchet motion," Physical Review Letters, vol. 99, pp. 247002 1-4, 2007.

[8] J. F. Wambaugh, C. Reichhardt, C. J. Olson, F. Marchesoni, and F. Nori, "Superconducting fluxon pumps and lenses," Physical Review Letters, vol. 83, no. 24, pp. 5106-5109, Dec. 1999.

[9] M. Magnasco, "Forced thermal ratchets," Physical Review Letters, vol. 71, no. 10, pp. 1477-1481, Sep. 1993.

[10] Y. Togawa, K. Harada, T. Akashi, H. Kasai, T. Matsuda, F. Nori, A. Maeda, and A. Tonomura, "Direct observation of rectified motion of vortices in a niobium superconductor," Physical Review Letters, vol. 95, pp. 087002 1-4, Aug 2005.

[11] G. W. Webb, "Low-temperature electrical resistivity of pure niobium," Physical Review, vol. 181, no. 3, pp. 1127-1135, 1969. 